\title{
VIDAS DE ACTIVISMO. LA PARTICIPACIÓN EN LOS MEDIOS DE COMUNICACIÓN COMUNITARIOS COMO FUENTE DE APRENDIZAJES
}

\author{
ACTIVIST LIVES. \\ PARTICIPATION IN COMMUNITY-BASED \\ MEDIA AS SOURCES OF LEARNING
}

Ángel Barbas Coslado* y Ana Martínez Ortiz de Zárate ${ }^{* *}$

\begin{abstract}
En este artículo se identifican y se analizan las experiencias de aprendizaje de personas que han participado en medios de comunicación comunitarios, con el fin de contribuir al conocimiento de este tipo de medios como entornos de aprendizaje. Nos basamos en el paradigma de la educomunicación y utilizamos el método biográfico para recopilar ciertas historias de vida que nos han permitido establecer una categorización compuesta por tres grandes dimensiones de aprendizaje: la cognitiva, la emocional y la sociopolítica. El objetivo último es mostrar la riqueza de dichos aprendizajes, valorar el impacto que estas experiencias han podido tener en las vidas de los participantes y poner de manifiesto el potencial pedagógico de los medios de comunicación comunitarios.

Palabras claves: Medios de comunicación comunitarios, educomunicación, historias de vida, experiencias de aprendizaje, dimensiones de aprendizaje.
\end{abstract}

In this paper we identify and analyse the learning experiences of people who have been involved in community-based media, in order to contribute to knowledge of this sort of media as learning environments. We rely on the ducommunication paradigm and use the biographical method to collect a set of life-story interviews which allow us to establish a compound categorization of three major areas of learning, which are: cognitive, emotional and sociopolitical. The final goal is to show the wealth of these learnings, to put in value the impact that such experiences have had on the lives of participants and to highlight the pedagogical potential of community-based media.

Key words: Community-based media, educommunication, life-stories interviews, learning experiences, learning dimensions.

\section{Introducción}

Los medios de comunicación comunitarios son espacios abiertos a la participación de la ciudadanía y las personas que se involucran en ellos conforman entornos de aprendizaje constituidos por redes de intercambio y prácticas sociales, mediáticas y culturales. Este artículo es fruto de una investigación que tiene como objetivo principal identificar las experiencias de aprendizaje de personas que han participado en este tipo de medios, así como analizar y categorizar dichas experiencias con el fin de contribuir a su conocimiento como entornos de aprendizaje. Enmarcamos nuestra investigación en el paradigma sociocrítico y en la tradición académica de la educomunicación dialógica.
Para ello, en primer lugar, exponemos el marco conceptual tomando como referencia el corpus teórico elaborado desde hace varias décadas acerca de los medios de comunicación comunitarios, así como los fundamentos de la educomunicación desde la epistemología latinoamericana.

En segundo lugar, detallamos el procedimiento metodológico utilizado para la recogida de información durante el trabajo de campo; argumentamos por qué hemos considerado a las historias de vida como la metodología más adecuada para lograr los objetivos planteados; establecemos los criterios utilizados para la selección de la muestra; y, finalmente, definimos las tres categorías de aprendizaje que hemos elaborado a partir del análisis de las entrevistas: 1) la cognitiva; 2) la emocional; y, 3) la sociopolítica.

\footnotetext{
* Universidad Nacional de Educación a Distancia (UNED), España. Correo electrónico: abarbas@madrid.uned.es

** Universidad Nacional de Educación a Distancia (UNED), España. Correo electrónico: azarat.ana@ gmail.com
} 
En tercer lugar, llevamos a cabo una discusión entre los testimonios más representativos de nuestros entrevistados y el corpus teórico de referencia; y hemos organizado dicha discusión basándonos en las tres categorías elaboradas durante el análisis de las entrevistas.

En las conclusiones argumentamos el potencial pedagógico de los medios de comunicación comunitarios, en la medida en la que las personas que participan en ellos desarrollan varias experiencias de aprendizaje que son útiles para la producción mediática y para la participación en este tipo de colectivos, pero también para otros ámbitos de la vida. Para finalizar, planteamos ciertos aspectos que consideramos necesarios para poder trazar líneas de investigación en la confluencia de los ámbitos científicos de la Comunicación y la Educación.

\section{Los medios de comunicación comunitarios como entornos de aprendizaje: un acercamiento desde la educomunicación}

Al hablar de medios de comunicación comunitarios estamos haciendo referencia a todos aquellos medios de comunicación producidos por organizaciones y colectivos sociales sin ánimo de lucro, así como organizaciones no gubernamentales (ONG y ONGD); asociaciones juveniles, culturales y vecinales, movimientos sociales, cooperativas y empresas de economía social, entre otros.

No existe consenso a la hora de definir este tipo de medios, pues cada experiencia se inserta en un contexto sociocultural determinado y se caracteriza, por tanto, por varios rasgos muy particulares (Gumucio, 2001). Sin embargo, existen algunos elementos comunes que nos permiten hablar de los medios comunitarios como un fenómeno social con entidad propia. Concretamente, se trata de experiencias que se caracterizan por: a) un modelo de comunicación dialógico y orientado hacia la crítica social (Barranquero y Meda, 2015); b) una filosofía no lucrativa y basada en el servicio a la comunidad para favorecer la cohesión social y el empoderamiento de la ciudadanía (Milan, 2010); c) una producción informativa que busca visibilizar colectivos, realidades y conflictos sociales que no suelen tener cabida en los medios convencionales (Huesca, 2007); d) la importancia de las interacciones sociales que se dan junto con la producción y circulación de contenidos (Gumucio, 2001); e) la participación amplia de la ciudadanía en todas las fases y actividades de este tipo de proyectos (Marí, 2010); f) un modelo de organización basado en una estructura horizontal y en un sistema asambleario de toma de decisiones (Santos, 2006a; Rodríguez, 2009); y, g) un sistema de financiación autogestionado que permite mantener la autonomía y la independencia (Gumucio, 2005).

Desde las experiencias paradigmáticas de la segunda mitad del siglo XX -especialmente las radios mineras bolivianas y las radios educativas en Colombia-, los medios de comunicación comunitarios y ciudadanos han demostrado ser un espacio fecundo para la creatividad, para el empoderamiento político, para la cohesión social, para la recuperación de la memoria histórica y para el reconocimiento y la puesta en valor de las identidades (Gumucio y Tufte, 2008).

Todos estos aspectos nos permiten afirmar que los medios de comunicación comunitarios tienen un claro potencial educativo por cuanto generan transformaciones en las personas que se involucran en ellos; y en la medida en que esas transformaciones se orientan a la mejora de las capacidades de comunicación de los participantes, así como a la ampliación de los marcos de interpretación que les permiten una toma de conciencia y la adquisición de repertorios de acción para una mayor implicación en la vida pública. Podríamos decir, por tanto, que desde la perspectiva de los aprendizajes informales los medios de comunicación comunitarios se configuran como escuelas de política (Barbas y Postill, 2017), entendiendo lo político desde el sentido etimológico de lo que tiene relación con la ciudadanía, pues ponen en práctica ciertas dinámicas en donde aprenden a desarrollar nuevos modelos afectivos, nuevas formas de relación e interacción entre unos y otros, entre los sujetos y la realidad, y entre los sujetos y sí mismos (Marí, 2011).

En el plano teórico, fundamentamos la perspectiva pedagógica de los medios de comunicación comunitarios en la tradición epistemológica de la educomunicación; un campo de estudios que tiene su origen en el pensamiento comunicacional latinoamericano y, más concretamente, en la filosofía del educador brasileño Paulo Freire, quien estableció por primera vez una relación de 
reciprocidad entre prácticas de comunicación y prácticas de educación, y concibió los procesos de comunicación/educación como círculos de cultura con los que se genera el aprendizaje de los participantes.

Las obras de Freire $(1965,1970)$, en primer lugar, y de autores como Gutiérrez $(1973,1975)$ y Kaplún (1985, 1992), posteriormente, permitieron establecer las bases de un modelo teórico de comunicación/educación, enraizado en las prácticas comunicacionales y educativas que se estaban llevando a cabo a nivel popular en numerosas poblaciones latinoamericanas, y cuyos objetivos fundamentales se pueden concretar en: la importancia de la participación, del empoderamiento y de la toma de conciencia de la ciudadanía para generar procesos de transformación a nivel político, social y cultural; en palabras de Freire, para impulsar procesos de concientización que permitan desarrollar procesos de politización (1970).

Este es el marco conceptual en el que hemos apoyado nuestro trabajo, el punto de partida teórico de esta investigación. No obstante, los propios datos recogidos durante el trabajo de campo, y su posterior análisis, nos han llevado a profundizar en planteamientos teóricos que no habíamos considerado en esta revisión bibliográfica inicial, como veremos en el apartado dedicado a la discusión de resultados.

\section{Metodología y análisis de datos}

Nuestro interés por identificar las experiencias de aprendizaje de personas que han participado en medios de comunicación comunitarios nos ha llevado a la utilización de las historias de vida como herramienta de investigación. Podemos definir las historias de vida como el "proceso de indagación, a través de una metodología fundamentada en entrevistas y charlas entre investigador y protagonista, sobre los sentimientos, la manera de entender, comprehender, experimentar y vivenciar el mundo y la realidad cotidiana" (Martín-García, 1995: 42).

La construcción de las historias de vida es un proceso de indagación de las personas en sus propias vidas, en diálogo con el investigador, de manera que puedan otorgar sentido a sus procesos de aprendizaje a la luz de sus relatos autobiográficos.
La elección de esta técnica de recogida de información ha estado motivada no solo por su grado de coherencia con los objetivos que nos hemos planteado, sino también por el enfoque adoptado en el desarrollo de nuestra investigación y por las características de la muestra que hemos utilizado para realizar las entrevistas. Por otra parte, nuestro enfoque entronca con las corrientes de investigación enraizadas en el saber popular (García Canclini, 1990). Nuestro objetivo implica resaltar la importancia del conocimiento generado por la propia comunidad, más allá de verdades absolutas o de planteamientos académicos ortodoxos; y, asimismo, poner en valor el conocimiento producido por las propias dinámicas sociales en las que se involucra la ciudadanía para la transformación y mejora de los entornos en los que se incardinan (Cortés, 2010). No sería posible identificar las experiencias de aprendizaje de las personas que participan en medios de comunicación comunitarios sin involucrarnos en la narración de sus propias vidas, sin sumergirnos en su visión del mundo, sin atender al relato que construyen de sus vivencias y del contexto sociocultural en el que se han desarrollado como activistas y como personas comprometidas con su entorno; y la historia de vida, como técnica de investigación, es un instrumento que sí nos permite este tipo de acercamiento a la realidad de los participantes.

Asimismo, la utilización de las historias de vida es coherente con el modelo de comunicación participativo y democrático de los medios comunitarios, en la medida en la que se trata de una técnica de recogida de información que concibe a las personas entrevistadas como sujetos activos y no como objetos pasivos en manos del investigador.

Por otra parte, muy relacionado con el enfoque de investigación que hemos adoptado, otro aspecto que consideramos muy relevante de las historias de vida es su capacidad de empoderar a los participantes que narran sus experiencias, ya que "cuando se narra una vida no solo se recoge lo que se ha aprendido en ella, sino que en el acto de narración mismo acontece un aprendizaje, es decir, la narración es un aprendizaje-en-acción" (Bolívar, 2014: 720).

Finalmente, las características de la muestra que hemos seleccionado para realizar nuestra investigación ha sido también un elemento 
importante para la elección de las historias de vida como técnica de recogida de información.

Nos hemos basado en tres criterios para la selección de los informantes: 1) la participación de las personas entrevistables en medios de comunicación comunitarios; 2) la percepción por parte de dichas personas de que su participación en medios de comunicación comunitarios ha sido lo suficientemente significativa como para considerar que estas experiencias han sido claves en sus aprendizajes desde el punto de vista cognitivo, emocional o sociopolítico; y, 3) su predisposición para realizar diferentes entrevistas en profundidad con las que transmitir sus vivencias mediante un relato autobiográfico.

A este respecto, es importante mencionar que nuestros informantes responden a un perfil muy heterogéneo, algo que también va en consonancia con el pluralismo interno y externo que caracteriza a los medios de comunicación comunitarios (Gumucio, 2001).

En nuestra investigación encontramos personas que participan en medios de comunicación comunitarios que usan diferentes formatos de producción de contenidos, personas que tienen niveles formativos diferentes, con orígenes diversos, y que se ubican en distintos espacios geográficos (Uruguay y España). Se han incluido testimonios recogidos en Uruguay, porque nuestros referentes teóricos, como hemos visto, se sitúan principalmente en América Latina. En concreto, Uruguay es un país con una importante tradición en comunicación comunitaria y en educomunicación, con importantes autores como Mario Kaplún. Actualmente, su hijo, Gabriel Kaplún, profesor de la Universidad de la República, continúa trabajando en este campo mediante la coordinación del grupo de investigación AlterMedia (Alternativas Mediáticas) junto con otros académicos como Alicia García, Martín Martínez, Lucía Olivari y Facundo Franco, entre otros.

Las personas entrevistadas pertenecen a los siguientes proyectos comunicativos comunitarios: Las radios Ágora Sol Radio y Radio Almenara, los periódicos Madrid 15-M y El Salto, la plataforma audiovisual Tomalatele (estos cinco proyectos situados en Madrid, España); y la radio comunitaria Vilardevoz, ubicada en Montevideo (Uruguay).

Hemos realizado 40 entrevistas semiestructuradas a personas que han estado implicadas en experiencias comunicativas comunitarias de tres formatos mediáticos diferentes (radio comunitaria, comunicación audiovisual participativa y los escenarios colaborativos virtuales). Con estas personas hemos llevado a cabo uno o dos encuentros para realizar entrevistas en profundidad. La duración media de cada entrevista ha sido alrededor de una hora, pero en muchos casos tenemos entrevistas de más de 2 horas de duración. En total, contamos con más de 80 horas de entrevistas grabadas en audio.

Las entrevistas han sido de tipo semiestructurado y en profundidad, basándonos en un guion formado por los siguientes ítems temáticos:

- Datos personales y contexto familiar.

- Experiencia en comunicación comunitaria (causas y motivaciones).

- Proceso de aprendizaje y transformación personal.

- Contexto, historia y evolución del medio de comunicación comunitario.

- Relación y transformación de la comunidad a partir del surgimiento de la experiencia de comunicación comunitaria.

Tras realizar las entrevistas se ha procedido a su transcripción y análisis utilizando el software Atlas.ti (V. 8); este análisis nos ha permitido identificar dimensiones clave de aprendizaje:

1. La dimensión cognitiva, donde englobamos los aprendizajes relativos a la ampliación de los marcos de interpretación y los esquemas mentales, en relación con la comprensión y análisis de la realidad.

2. La dimensión emocional, en la que recogemos los aprendizajes relativos al autoconocimiento y al desarrollo personal, a la comprensión de las emociones de los demás y al manejo de las emociones propias.

3. La dimensión social y política, con la que hacemos referencia a los aprendizajes procedimentales, basados en los aprendizajes emocionales y cognitivos, que son necesarios para participar de forma activa en un medio de comunicación comunitario.

\section{Discusión}

La discusión teórica se establece a partir de los testimonios más representativos de nuestros 
entrevistados y tomando como referencia un marco conceptual formado por diferentes estudios que nos permiten profundizar en los aspectos cognitivos, emocionales y sociopolíticos detectados. La propuesta es elaborar un análisis interpretativo que nos permita teorizar acerca de la relevancia de los medios de comunicación comunitarios como entornos de aprendizaje.

\section{Dimensión cognitiva}

La teoría de los esquemas de interpretación o marcos (frames) desarrollada por Bateson (1972) y Goffman (1974) nos sirve de base para analizar los aprendizajes que hemos categorizado bajo la denominación de "dimensión cognitiva". Los marcos de interpretación son esquemas que permiten a los individuos localizar, percibir, identificar y categorizar hechos del mundo en general; son estructuras mentales y culturales que permiten el reconocimiento del mundo, dar sentido a la realidad, guiar la percepción y canalizar la acción (Della Porta y Diani, 2011).

Los esquemas o marcos de interpretación son flexibles y cambiantes debido a la plasticidad cerebral. Las personas tomamos como referencia nuestros esquemas o marcos de interpretación para pensar, actuar y tomar decisiones; y modificamos nuestros esquemas o marcos de interpretación a medida que interactuamos con nuestro entorno y a medida que vamos desarrollando aprendizajes (Goffman, 1974; Snow et al., 1986).

Cuando las personas se implican y participan activamente en un determinado proyecto experimentan importantes cambios en diferentes niveles. Sus marcos de referencia se modifican, sus percepciones iniciales acerca de una realidad pueden cambiar, e incluso pueden llegar a descubrir otra nueva realidad que hasta entonces había permanecido escondida, generándose, por tanto, otros marcos de referencia (Goffman, 1974; Hunt, Benford y Snow, 2001).

Algunas de las experiencias narradas por nuestros entrevistados muestran cómo sus esquemas mentales previos y sus marcos de interpretación pudieron haberse transformado a raíz de participar en un proyecto de comunicación comunitaria. Por ejemplo, dos de ellas han reconocido un cambio en relación con los prejuicios y al desconocimiento que tenían hacia determinados colectivos y problemáticas sociales. La informante 1 (Radio Almenara), logró vencer algunos prejuicios que tenía hacia determinados colectivos y luchas sociales, algo que le permitió transformar sus esquemas previos y comprender mucho mejor las luchas de movimientos como el movimiento okupa; y el informante 2 (Tomalatele), considera que su participación en este colectivo le ha hecho "conocer realidades, empatizar con situaciones que antes veía de otra manera, y abrir mucho la mente y la conciencia".

En el mismo sentido, la informante 3 (Ágora Sol Radio) narra cómo ha cambiado su identidad política, algo que está muy relacionado con la capacidad para entender los aspectos sociales en toda su complejidad y no quedarse en el prejuicio, en el estereotipo o en la adopción acrítica del discurso propuesto por una determinada corriente ideológica o partido político: "Mi identidad política ha cambiado mucho en los últimos años, especialmente desde que estoy participando en Ágora Sol Radio, y eso también ha hecho que cada vez me resulte más difícil definirme".

Por su parte, el informante 4 (Diagonal y $E l$ Salto), que proviene de una familia que él mismo define como "burguesa", y que nunca tuvo ningún tipo de relación con el mundo de la política ni del activismo, dice que comenzó a participar en medios comunitarios a partir de la aparición del Movimiento 15-M y que esto supuso un antes y un después en su manera de ver el mundo y de verse a sí mismo:

Yo antes tenía mucha necesidad de hablar de mí a través de mi trabajo, pero [cuando empiezo a trabajar en medios de comunicación comunitarios], empiezo a ver que es mucho más importante hablar de lo que nos pasa a todos. No es solo un cambio de enfoque profesional sino que es un cambio personal muy profundo, porque dejo de estar preocupado por lo que a mí me pasa y me doy cuenta de que más allá de mis 'pajas mentales' hay un tipo al que lo van a dejar en la calle mañana, un tipo que no tiene dónde dormir, y creo también que esto ha sido una forma de maduración [...]. Porque yo venía de un entorno bastante burgués, o sea, un entorno que no era el más adecuado para darme cuenta de cómo funcionaban las 
cosas a otro nivel, en otros sitios... y claro, esto me ha cambiado mucho.

De igual modo, el informante 2 (Tomalatele) reconoce que ha cambiado su concepción de la política a raíz de su participación en medios de comunicación comunitarios:

Yo en política no había estado, digamos metido para nada, bueno, sí que lo había estado, pero de otra manera, porque al final, he descubierto que todo es política. Cómo organizas tu vida, eso también es política. Del sistema surge el contrasistema por cosas que no cuadran, que todo es un negocio, que está montado todo para el negocio. Por y para el negocio, sin mirar por la salud. Al final, es política, pero de otra manera.

Estos testimonios ejemplifican cómo la participación en medios de comunicación comunitarios puede influir en la transformación de los marcos de interpretación a los que hacíamos alusión anteriormente. Pero este tipo de aprendizajes que hemos categorizado bajo la denominación de "cognitivos", como veremos a continuación, están estrechamente relacionados con la dimensión emocional.

\section{Dimensión emocional}

Las experiencias de aprendizaje emocional se producen y evolucionan a la par que los aprendizajes cognitivos, pues cada marco cognitivo implica la creación de un marco emocional y viceversa. Por tanto, la distinción que hacemos entre dimensión cognitiva y dimensión emocional es una distinción artificial con el objetivo de estructurar de forma coherente el presente texto y establecer diferencias conceptuales entre unos y otros tipos de aprendizajes; pero, así como indica Damasio (2011), no se puede separar la mente del cuerpo, ni el pensamiento de las emociones.

La relación existente entre las emociones y los movimientos sociales ha sido estudiada por diversos autores (Goodwin, Jasper y Polletta, 2001; Flam y King, 2005; Della Porta, 1998; Jasper, 2012); una tendencia que forma parte del denominado "giro afectivo" de la investigación en Ciencias Sociales (Lara y Enciso, 2013).
Los movimientos sociales ya no solo actúan hacia el exterior, sino que también trabajan hacia el interior de los mismos participantes, integrando la vida afectiva en la acción transformadora (Corominas, 2002). Esto es algo que también hemos comprobado en nuestras entrevistas; por ejemplo, la informante 5 (Ágora Sol Radio) hacía muchas referencias al cuidado del otro como estrategia política, a la importancia de cuidarse los unos a los otros por el bien del colectivo y por el bien de cada uno de sus miembros; los cuidados, por tanto, son un aspecto central y estratégico para la construcción de su identidad y para el desarrollo de sus acciones.

En este sentido, tomamos como referencia el trabajo elaborado por Gilligan (1985) pertinente a la ética del cuidado. Según esta autora, la ética del cuidado se apoya en la premisa de la no violencia y se manifiesta cuando se crean "redes de relaciones con ternura y responsabilidad, sin que sea necesario destruir el propio yo" (p. 281).

La informante 1 (Radio Almenara) nos muestra la importancia que tiene para ella la ética de los cuidados; algo que ha aprendido a tener en cuenta a partir de su implicación en la experiencia comunitaria de Radio Almenara. También la informante 6 (Vilardevoz) reconoce este aprendizaje:

Aprendí a que me cuidaran y me quisieran. Gracias a todos ellos aprendí a cuidar y a amar, a querer a los otros [...]. Para ser yo, una persona a la que antes le importaba todo un huevo..., una persona que no le importaba a nadie, que pensaba en ella, que siempre era ella, ella, ella..., entonces entre otras cosas aprendí todo esto de los cuidados.

Pero la importancia que nuestros entrevistados otorgan a esta dimensión emocional se pone especialmente de manifiesto desde la autocrítica, cuando reconocen sus carencias en educación emocional a la hora de resolver algunos de los conflictos interpersonales que se dan en sus dinámicas cotidianas:

[...] hay problemas que nunca terminamos de resolver por muchas asambleas que hagamos, y es que yo creo que nos falta mucha educación emocional en el sentido de reconocer las emociones 
propias y las de los otros, y a partir de esa empatía relacionarnos de otra manera para enriquecernos con nuestras diferencias; eso sería lo ideal para que el colectivo avance, pero también es importante para que cada uno de nosotros pueda aprender" (Informante 7, Madrid 15-M).

En la misma línea, la informante 8 (Vilardevoz) considera necesaria una educación profunda y de forma continuada en este aspecto:

[...] la gente no sabe a veces ni qué es quererse; piensan que quererse a uno mismo es algo equivocado, si creciste en la crítica y en la exigencia, para vos quererte es eso. Entonces poder tener otras voces u otros diálogos te ayuda a decir: "pero entonces... hay algo que tengo que cambiar; yo pensé que me estaba cuidando, pero no me estoy cuidando y tampoco estoy cuidando al otro".

Los aprendizajes emocionales y cognitivos son necesarios para participar de forma activa en cualquier ámbito; y, en este sentido, son la base para conocer los procedimientos sociopolíticos implicados en un medio de comunicación comunitario y actuar conforme con dichos procedimientos. Podríamos decir, en suma, que sin estos aprendizajes emocionales y cognitivos no podría darse una participación plena, y que son dichos aprendizajes el fundamento esencial para poder implicarse y formar parte de este tipo de colectivos.

\section{Dimensión sociopolítica}

Los proyectos de comunicación comunitarios se caracterizan porque los miembros se relacionan de una forma horizontal y se organizan siguiendo metodologías asamblearias (Santos, 2006a). No obstante, estas formas organizativas requieren de varios aprendizajes previos entre los que se encuentran aprendizajes de tipo emocional y cognitivo, que son el fundamento para los aspectos procedimentales implicados en la toma de decisiones.

Entendemos la horizontalidad como la relación no jerárquica, lejos de la dependencia y del dominio; y también como la actitud mental que resulta imprescindible para tener un diálogo auténtico con las otras personas y para emprender proyectos colectivos (Santos, 2006b). En nuestro trabajo de campo hemos identificado que la horizontalidad no solo es una meta, sino que es la herramienta para conseguir los objetivos del colectivo (Sitrin, 2005).

La informante 8 (Radio Vilardevoz) señalaba lo siguiente al respecto:

El espacio más importante de Vilardevoz es la asamblea, porque ahí se decide todo. A dónde vamos; para qué se utiliza el dinero; qué podemos hacer para apoyar a un compañero que tiene un problema. Entonces, también la asamblea es como una terapia, digamos nosotros, porque tomar decisiones de esta manera es bueno para todos.

No obstante, aunque los movimientos de comunicación comunitaria se caracterizan por sus relaciones horizontales y por tomar decisiones siguiendo la metodología asamblearia, esto no está exento de conflictos ni del peligro de que surjan relaciones de poder, grupos autoritarios o personas dominantes (Ferree y Roth, 1998; Mata, 2015).

El informante 2 (Tomalatele) ha señalado problemas relacionados con choques de egos entre miembros del grupo:

[...] el ser humano es el ser humano y los conflictos de egos surgen en todas partes. Yo he conocido colectivos y un montón de situaciones, y he estado en asambleas donde he visto problemas y conflictos por cuestiones de egos.

Decidir de forma asamblearia y horizontal también tiene más obstáculos. Martínez y Lorenzo (2005) señalan algunos como que las asambleas sean muy largas y poco productivas, que se desarrollen de forma poco participativa o incluso antidemocrática o que resulten pesadas y poco atrayentes. Pero nosotros queremos destacar especialmente cómo algunos de nuestros entrevistados manifiestan una vez más una toma de conciencia respecto de sus carencias organizativas y la necesidad de aprender a partir de la teoría y de la práctica: 
La asamblea me parece el mejor órgano político para la autogestión y el autogobierno pero en las asambleas también se reproducen roles y poderes... ¿qué diferencia hay entre la manipulación de un partido político y la manipulación del que tiene más carácter o del que habla mejor en la asamblea? El asamblearismo no es solo buena voluntad, tiene toda una teoría y unos procedimientos detrás que también son particulares de cada sitio, tiene una serie de elementos que hacen que si algo falla sea porque no hemos aplicado bien las teorías al contexto concreto, porque no hemos aprendido a hacerlo mejor (Informante 9, Madrid 15-M).

La metodología asamblearia requiere práctica y entrenamiento, pues las personas no tienen conocimientos suficientes para participar en una asamblea; y, además, el medio social dominante favorece otras formas de organización, menos horizontales y más jerarquizadas (Saravia, 2012; Mata, 2015).

Este aprendizaje implica una reeducación en términos de relaciones y en términos políticos, y fomenta el empoderamiento de las personas involucradas (Perkins y Zimmerman, 1995; Saravia, 2012; Mata, 2015; Johnson, 2015). Mediante el empoderamiento, los individuos o grupos reconocen tener el control de sí mismos y de los medios que necesitan para emprender acciones orientadas al desarrollo de capacidades nuevas y a la superación de situaciones problemáticas (Hernández y Martínez, 2006; Sánchez y Jaurena, 2015).

Para Perkins y Zimmermann (1995), el empoderamiento es la adquisición de una comprensión crítica del entorno, algo muy relacionado con las dimensiones cognitiva y emocional del aprendizaje. Y esa apropiación o empoderamiento se manifiesta también en acciones encaminadas a generar la toma de conciencia entre la ciudadanía, un aspecto que ha sido abordado en otros trabajos y definido según el principio de 'acción pedagógica' (Barbas y Postill, 2017). Por ejemplo, el informante 2 explica de esta forma el principal objetivo de Tomalatele:

Pretendemos ser algo que pueda empoderar a la gente, que haga la comunicación bidireccional, que empodere a la gente ya sea con concientización, mediante formación, mediante dar medios, mediante informar, hacer que la gente pueda pasar sus propios contenidos... Pues eso, un empoderamiento, que la gente misma pueda contar lo que está viendo.

Y por ese motivo, desde Tomalatele se organizan ciclos o actividades con la perspectiva de ampliar sus círculos; algo que también expresa la informante 1 (Radio Almenara):

Lo que estamos defendiendo con nuestro proyecto "Magnolias de Acero. Mujeres ilustres de Tetuán" es [...], empoderar a las mujeres del barrio. Que siempre se cuentan las historias de la reina de no sé dónde, de la Preysler, y bla, bla... y aquí, en nuestro barrio, hay mujeres que tienen unas historias que son para enmarcar. Y eso es un poco lo que queremos resaltar y destacar.

Sin embargo, los entrevistados reconocen que aun llevando a cabo todos estos intentos, este tipo de proyectos de comunicación comunitaria siguen siendo desconocidos por la gran mayoría de la sociedad; como pone de manifiesto la investigación realizada por Ramos, Morais y Barranquero (2018). Nuestro informante 2 (Tomalatele) expresa esta idea de la siguiente manera:

Veo que somos islas incomunicadas o comunicadas de muy pequeña manera, de forma irregular, con listas de correo; que difundimos de forma conjunta mucho contenido y ya está. Pero que no hay colaboración real en grandes medios, aunque hemos hecho intentos.

\section{Conclusiones}

En este artículo hemos presentado parte de una investigación que busca explorar las relaciones que se establecen entre medios de comunicación comunitarios y procesos de aprendizaje. Más concretamente, hemos pretendido a identificar y categorizar las experiencias de aprendizaje de personas que han participado en este tipo de medios mediante la recopilación y análisis de sus relatos autobiográficos. El objetivo último es poner 
de manifiesto la riqueza de estas experiencias de aprendizaje y, en última instancia, contribuir al conocimiento del potencial pedagógico de los medios de comunicación comunitarios.

Participar activamente en un medio de comunicación comunitario genera importantes aprendizajes que pueden llegar a ser muy relevantes para la vida de las personas que se involucran en ellos. Nuestros informantes manifiestan importantes cambios en sus marcos de interpretación y esquemas mentales a raíz de su participación en este tipo de medios. Estos cambios cognitivos se concretan en el conocimiento de realidades que antes desconocían, en la reconfiguración de su identidad política y en la toma de conciencia acerca de la realidad social en general y respecto de su capacidad para la participación -por medio de su actividad en sus medios de comunicaciónen particular.

Asimismo, nuestros entrevistados hacen referencia a los aprendizajes emocionales que han adquirido, pues consideran que hay que trabajar hacia el interior de los colectivos cuidando la vida afectiva e integrándola en sus prácticas mediáticas y en sus dinámicas cotidianas. A este respecto, es importante mencionar la autocrítica que manifiestan cuando reconocen sus carencias en educación emocional a la hora de resolver algunos de los conflictos interpersonales que se dan en sus colectivos; algo que también pone de relieve la toma de conciencia que han desarrollado respecto de la importancia que los aspectos emocionales tienen en este tipo de proyectos de comunicación.

No obstante, como hemos argumentado, existe una estrecha relación entre la dimensión cognitiva y la dimensión emocional; pues son facultades mentales que se complementan y se refuerzan mutuamente, y que juegan un papel esencial en el conocimiento procedimental necesario para la participación activa y, en general, para el desarrollo de las capacidades requeridas a la hora de acometer acciones concretas e involucrarse en las dinámicas sociales y organizativas del día a día.

En este sentido, los aprendizajes cognitivos y emocionales son la base de los aprendizajes procedimentales necesarios para la participación social plena; y son asimismo aprendizajes fundamentales para participar en entornos caracterizados por relaciones horizontales y por dinámicas asamblearias. En relación con esto, nuestros entrevistados relataban cómo su participación en colectivos de comunicación comunitaria les ha permitido aprender dichos procedimientos y también generar una toma de conciencia acerca de la necesidad de tener una formación específica en estos aspectos.

En síntesis, hemos constatado la riqueza y la relevancia de los aprendizajes que nuestros entrevistados han logrado mediante su participación en medios de comunicación comunitarios; algo que ellos mismos ponen de manifiesto cuando señalan cómo estos aprendizajes les han servido, no solo para participar activamente en este tipo de colectivos, sino también para desarrollarse como personas al margen de su militancia política o de su identidad activista. Consideramos, por tanto, que los medios de comunicación comunitarios son entornos con un gran potencial para generar experiencias de aprendizaje caracterizadas por su riqueza -en tanto que es posible aprender aspectos cognitivos, emocionales y procedimentales-, y caracterizadas por su relevancia -en tanto que son útiles para participar en medios comunitarios pero también en otras esferas de la vida-.

Esta constatación nos ha permitido esbozar varios aspectos que consideramos necesarios para poner en valor el potencial pedagógico de los medios de comunicación comunitarios y para trazar líneas de investigación que nos permitan seguir avanzando en el conocimiento de este tipo de medios como entornos de aprendizaje, estos son: 1) que los procesos de aprendizaje son mucho más significativos cuando la motivación y las emociones juegan roles importantes; 2) que la identificación con determinados valores compartidos y procedimientos promueve la implicación de los participantes; 3) que el reconocimiento de unos objetivos grupales como objetivos propios es un factor que moviliza la acción; 4) que la acción en colaboración incrementa el autoconocimiento y la asunción de responsabilidades; y, 5) que los lazos afectivos que se establecen a base de unos objetivos comunes crean espacios de confianza que pueden ser fundamentales para que tengan lugar determinadas experiencias de aprendizaje.

Concluimos con la propuesta de utilizar estos aspectos como principios teóricos sobre los que apoyar los estudios de Educación Social en general, y las investigaciones de la educación no formal e informal desde la perspectiva de la educomunicación en particular. 


\section{Agradecimientos}

Este artículo forma parte de las líneas de trabajo desarrolladas por la Red de Investigación en Comunicación Comunitaria, Alternativa y Participativa (RICCAP) de España. Los autores, miembros de dicha red, manifiestan su agradecimiento a los colegas participantes en la misma, así como a la Universidad Nacional de Educación a Distancia (UNED) por su apoyo institucional, y a las personas y colectivos que de una u otra manera lo han hecho posible.

\section{Referencias Citadas}

Barbas, A. \& Postill, J.

2017 Communication Activism as a School of Politics: Lessons from Spain's Indignados Movement. Journal of Communication, 67 (5): 646-664.

Barranquero, A. \& Meda, M.

2015 Los medios comunitarios y alternativos en el ciclo de protestas ciudadanas desde el 15M. Athenea Digital, 15 (1): $139-170$

Bateson, G.

1972 Steps to an Ecology of Mind. Collected essays in Anthropology, Psychiatry, Evolution and Epistemology. The University of Chicago Press, Chicago.

Bolívar, A

2014 Las historias de vida del profesorado. Voces y contexto. Revista Mexicana de Investigación Educativa, 19 (62): 711-734

García Canclini, N.

1990 Culturas Híbridas. Estrategias para Entrar y Salir de la Modernidad. Grijalbo, México.

Corominas, J.

2002 La acción humana. Reproducción y transformación del sistema social mundial, www.uca.edu.sv/facultad/ chn/c1170/laaccionhumana.html (2 febrero 2019).

Cortés, M.I.

2010 Aproximaciones epistemológicas al concepto de comunicación comunitaria. Revista de Investigaciones Desbordes, 1: 77-80.

Damasio, A.

2011 El Error de Descartes. Destino, Barcelona.

Della Porta, D.

1998 Las motivaciones individuales en las organizaciones políticas clandestinas. En Los Movimientos Sociales. Transformaciones Políticas y Cambio Cultural, editado por P. Ibarra y B. Tejerina, pp. 219-242. Trotta, Madrid.

Della Porta, D. \& Diani, M.

2011 Los movimientos Sociales. CIS y Editorial Complutense, Madrid.

Ferrée, M. \& Roth, S.

1998 Gender, Class and the Interaction among Social Movements: A Strike of West Berlin Daycare workers, Gender and Society, 12 (6): 626-648

Flam, H. \& King, D. (eds.)

2005 Emotions and Social Movements. Routledge, London. Freire, P.

1965 La Educación como Práctica de la Libertad. Siglo XXI, Santiago de Chile.

Freire, P.

1970 Pedagogía del Oprimido. Siglo XXI, Buenos Aires.
Gilligan, C.

1985 La Moral y la Teoría: Psicología del Desarrollo Femenino. Fondo de Cultura Económica, México D.F.

Goffman, E.

1974 Frame Analysis. An Essay on the Organization of Experience. Northeastern University Press, Boston.

Goodwin, J.; Jasper, J. \& Polletta, F. (eds.).

2001 Passionate Politics: Emotions in Social Movements. University of Chicago Press, Chicago.

Gumucio, A.

2001 Haciendo Olas: Historias de Comunicación Participativa para el Cambio Social, The Rockefeller Foundation, New York.

Gumucio, A.

2005 Arte de equilibristas: la sostenibilidad de los medios de comunicación comunitarios, Punto Cero, 10 (10): 6-19.

Gumucio, A. \& Tufte, T. (compil.)

2008 Antología de Comunicación para el Cambio Social: Lecturas Históricas y Contemporáneas. Consorcio de Comunicación para el Cambio Social/Plural Editores, South Orange (New Jersey), La Paz (Bolivia).

Gutiérrez, F.

1973 El Lenguaje Total. Una Pedagogía de los Medios de Comunicación. Humanitas, Buenos Aires.

Gutiérrez, F.

1975 Pedagogía de la Comunicación. Humanitas. Buenos Aires.

Hernández, J. A. \& Martínez, B.

2006 Género, empoderamiento y movimientos sociales: la Unión Campesina Emiliano Zapata Vive, en la región Tepeaca-Tecamachalco, Puebla. Región y sociedad, 18 (36): 107-146.

Huesca, R.

2007 Siguiendo el rastro de la historia de los enfoques de Comunicación Participativa en Desarrollo: Un acercamiento crítico. Redes.Com. Revista de Estudios para el Desarrollo Social de la Comunicación, 4: 21-42.

Hunt, S.; Benford, R. \& Snow, D.

2001 Marcos de acción colectiva y campos de identidad en la construcción social de los movimientos, en Los nuevos movimientos sociales. De la ideología a la identidad, editado por E. Laraña, y J. Gusfield, pp. 221-249. CIS, Madrid.

Jasper, J.

2012. Las emociones y los movimientos sociales: veinte años de teoría e investigación. Revista Latinoamericana de Estudios sobre Cuerpos, Emociones y Sociedad 10 (4): 48-68 
Johnson, D.

2015 Formar ciudadanos interculturales en un mundo global: algunas notas desde los estudios curriculares. Revista Diálogo Andino, 47: 7-14.

Kaplún, M.

1985 El Comunicador Popular. CIESPAL/CESAP/RADIO NEDERLAD, Quito.

Kaplún, M.

1992 A la Educación por la Comunicación. Unesco/ Orealc, Santiago de Chile.

Lara, A. y Enciso, G.

2013 El Giro Afectivo. Athenea Digital, 13 (3): 101-119.

Marí, V. M.

2010 El enfoque de la comunicación participativa para el desarrollo y su puesta en práctica en los medios comunitarios. Razón y Palabra, 15 (71): 1-18.

Marí, V. M.

2011 Comunicar para Transformar, Transformar para Comunicar. Tecnologías de la Información desde una Perspectiva de Cambio Social. Editorial Popular, Madrid.

Martín García, A.V.

1995 Fundamentación teórica y uso de las historias y relatos de vida. Aula, 7: 41-60.

Martínez, M. \& Lorenzo, A. R.

2005 Asambleas y reuniones. Metodologías de autoorganización. Traficantes de sueños, Madrid.

Mata, P.

2015 "Dos ojos o más". Liderazgos colectivos en el marco de la acción de una ciudadanía transformadora. Diálogo Andino, 47: 115-121.

Milan, S.

2010 Medios comunitarios y regulación. Una perspectiva de comunicación para el desarrollo. Investigación y Desarrollo, 14 (2): 268-291.

Montero, D. \& Moreno, J.M.

2014 El Cambio Social a través de las Imágenes. Guía para Entender y Utilizar el Vídeo Participativo. Catarata, Madrid.
Perkins, D. \& Zimmerman, M.A.

1995 Empowerment theory, research, and application. American Journal of Community Psychology, 23 (5): 569-579.

Pujadas, J.J.

2000 El método biográfico y los géneros de la memoria. Revista de Antropología Social, 9: 127-158.

Ramos, J.; Morais, S. \& Barranquero, A.

2018 Las redes de comunicación alternativa y ciudadana en España. Potencialidades, dificultades y retos. OBETS. Revista de Ciencias Sociales, 13 (1): 121-148.

Rodríguez, C.

2009 De medios alternativos a medios ciudadanos: Trayectoria teórica de un término. Folios. Revista de la Facultad de Comunicación de la Universidad de Antioquía, 21-22: 13-25.

Sánchez, H. y Jaurena, I.

2015 Análisis interseccional y enfoque intercultural en el estudio de la ciudadanía y la participación: consideraciones epistemológicas. Revista Diálogo Andino, 47: 143-149.

Santos, M.

2006a. De la verticalidad a la horizontalidad. Reflexiones para una educación emancipadora. Realidad: Revista de Ciencias Sociales y Humanidades, 107: 39-64.

Santos, $\mathrm{M}$

2006b. La horizontalidad de las relaciones humanas y la tolerancia. Utopía y praxis latinoamericana. Revista internacional de filosofía iberoamericana y teoría social, 11 (34): 79-90.

Saravia, $\mathrm{P}$.

2012 Movimientos sociales en Andalucía. Una mirada exploratoria a los movimientos críticos con la globalización neoliberal. Tesis doctoral. Departamento de Sociología, Universidad de Granada, Granada.

Sitrin, M. (ed.)

2005 Horizontalidad: Voces de Poder Popular en Argentina. Chilavert, Buenos Aires.

Snow, D.; Rochford, Jr.; Worden, S. K. \& Benford, R.

1986 Frame Alignment Process, Microbilization and Movement Participation. American Sociological Review, 51 (4): 464-481. 
